\title{
Equilibrium Point Bifurcation and Singularity Analysis of HH Model with Constraint
}

\author{
Xinhe $\mathrm{Zhu}^{1,2}$ and Zhiqiang $\mathrm{Wu} \mathrm{u}^{1}$ \\ ${ }^{1}$ Department of Mechanics, School of Mechanical Engineering, Tianjin University, Tianjin 300072, China \\ ${ }^{2}$ Department of Mathematics, School of Science, Tianjin Polytechnic University, Tianjin 300387, China
}

Correspondence should be addressed to Zhiqiang Wu; zhiqwu@tju.edu.cn

Received 10 April 2014; Accepted 23 June 2014; Published 7 July 2014

Academic Editor: Rehana Naz

Copyright ( $2014 \mathrm{X}$. Zhu and Z. Wu. This is an open access article distributed under the Creative Commons Attribution License, which permits unrestricted use, distribution, and reproduction in any medium, provided the original work is properly cited.

\begin{abstract}
We present the equilibrium point bifurcation and singularity analysis of $\mathrm{HH}$ model with constraints. We investigate the effect of constraints and parameters on the type of equilibrium point bifurcation. HH model with constraints has more transition sets. The Matcont toolbox software environment was used for analysis of the bifurcation points in conjunction with Matlab. We also illustrate the stability of the equilibrium points.
\end{abstract}

\section{Introduction}

The Hodgkin-Huxley nonlinear model (HH) [1] is one of the biggest challenges in the life science in the near history. $\mathrm{HH}$ quantitatively describes the electrical excitations of squid giant axon. Under the $\mathrm{HH}$ formalism, many mathematical models (HH-type) for diverse neurons are established [25]. A bifurcation is a qualitative change in the behavior of a nonlinear dynamical system as its parameters pass through critical values [6]. The study of bifurcations in neural models is important to understand the dynamical origin of many neurons and the organization of behavior. Many studies have been done on the bifurcation analysis of HH model. Guckenheimer and Labouriau [7] give the detailed bifurcation diagrams of $\mathrm{HH}$ model in two-parameter space of $I$ and $V_{\mathrm{K}}$. Bedrov reveals the possible bifurcations with changes of $g_{\mathrm{Na}}$ and $g_{\mathrm{K}}$, representing the maximal conductance of sodium and potassium, respectively $[8,9]$. The global structure of bifurcations in multiple-parameter space of the HH model is examined [10], and the details of the degenerate Hopf bifurcations are analyzed using the singularity theoretic approach [11]. Singularity theory offers an extremely useful approach to bifurcation problems [12]. The aim of this paper is to illustrate how constraints and parameters affect the dynamics of $\mathrm{HH}$ model. In the first attempt we choose $I$ as bifurcation parameter, $g_{\mathrm{K}}, g_{L}$ as unfolding parameters, and we restrict $V>0$; then we use the singularity theory of bifurcations and the computing method of bifurcations with constraint to obtain the new constraint transition sets. Secondly, using the above results, we investigate the effect of constraint and parameters on the type of equilibrium point bifurcation, and we also illustrate the stability of the equilibrium points.

\section{Hodgkin-Huxley Equations}

The HH comprises the following differential equations:

$$
\begin{aligned}
& \frac{d V}{d t}=\frac{1}{C_{M}}\left[I-g_{\mathrm{Na}} m^{3} h\left(V-V_{\mathrm{Na}}\right)\right. \\
& \left.\quad-g_{\mathrm{K}} n^{4}\left(V-V_{\mathrm{K}}\right)-g_{l}\left(V-V_{l}\right)\right], \\
& \frac{d m}{d t}=\alpha_{m}(1-m)-\beta_{m} m, \\
& \frac{d h}{d t}=\alpha_{h}(1-h)-\beta_{h} h, \\
& \frac{d n}{d t}=\alpha_{n}(1-n)-\beta_{n} n .
\end{aligned}
$$

$V$ represents the membrane potential. $0 \leq m \leq 1$ and $0 \leq h \leq 1$ are the gating variables representing activation 


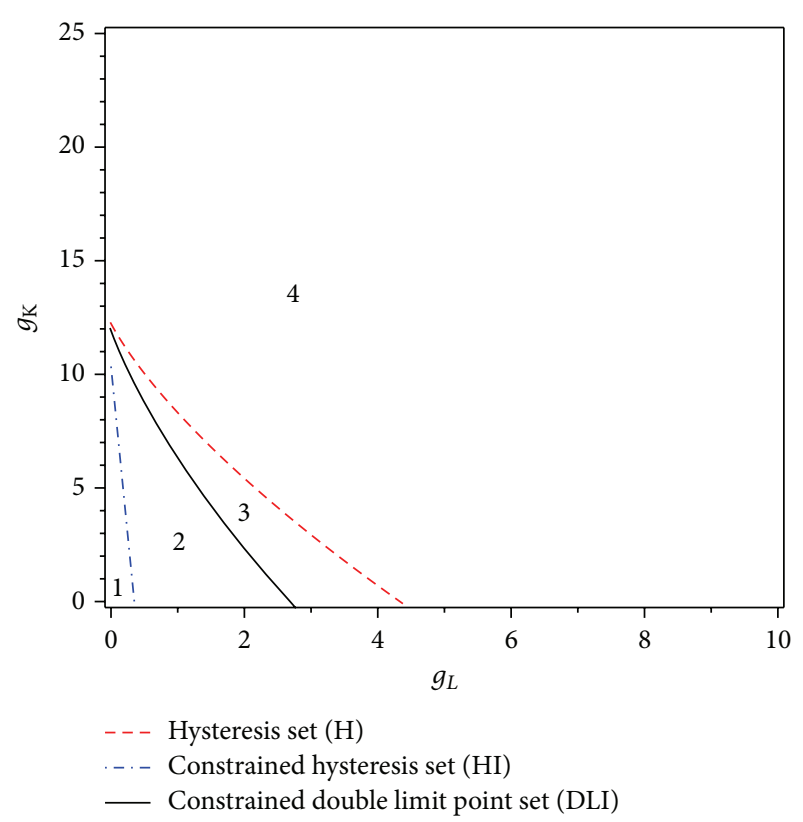

FIgURE 1: Constraint transition set.

and inactivation of the $\mathrm{Na}^{+}$current, respectively. $0 \leq n \leq 1$ is the gating variable representing activation of the $\mathrm{K}^{+}$current. $\alpha_{m}, \beta_{m}, \alpha_{h}, \beta_{h}, \alpha_{n}, \beta_{n}$ are the function of $V$ as follows:

$$
\begin{aligned}
& \alpha_{m}=\frac{2.5-0.1 V}{[\exp (2.5-0.1 V)-1]}, \quad \beta_{m}=4 \exp \left(\frac{-V}{18.0}\right), \\
& \alpha_{h}=0.07 \exp \left(\frac{-V}{20}\right), \quad \beta_{h}=\frac{1}{[\exp (3-0.1 V)+1]}, \\
& \alpha_{n}=\frac{(0.1-0.01 V)}{[\exp (1-0.1 V)-1]}, \quad \beta_{n}=0.125 \exp \left(\frac{-V}{80}\right) .
\end{aligned}
$$

The $\mathrm{HH}$ includes the following parameters: $V_{\mathrm{K}}=-12.0 \mathrm{mV}$, $V_{\mathrm{Na}}=115.0 \mathrm{mV}$, and $V_{l}=10.599 \mathrm{mV}$ representing the equilibrium potentials of $\mathrm{K}^{+}, \mathrm{Na}^{+}$, and leak currents, respectively. They are determined uniquely by the Nernst equation. $g_{\mathrm{Na}}=120.0 \mathrm{mS} / \mathrm{cm}^{2}, g_{\mathrm{K}}=36.0 \mathrm{mS} / \mathrm{cm}^{2}, g_{l}=$ $0.3 \mathrm{mS} / \mathrm{cm}^{2}$ represent the maximum conductance of the corresponding ionic currents. $C_{m}=1.0 \mu \mathrm{F} / \mathrm{cm}^{2}$ is the membrane capacitance. $I$ represents the external current, in $\mu \mathrm{A} / \mathrm{cm}^{2}$.

\section{Constrained Bifurcation Theory}

For the following bifurcation equation:

$$
g(u, \lambda ; \alpha)=0
$$

where $u, \lambda, \alpha$ are state variable, bifurcation parameter, and auxiliary parameter (or unfolding parameter), respectively. The bifurcation equation can deal with the singularity theories developed by Golubitsky and Schaeffer [12]. However, in some case, the variation of the state variable is often subjected to restriction, here called constraint. The forms of constraints are different in different problems, of which the most popular single-sided constraint is listed here [13].

The mathematical expression with single-sided constraint is

$$
\begin{aligned}
& g(u, \lambda ; \alpha)=0, \\
& \delta[u-U] \geq 0 .
\end{aligned}
$$

The following are transition sets for single-sided constraint:

$$
\begin{aligned}
& \text { (B) : }\left\{\begin{array}{c}
g(u, \lambda ; \alpha)=0 \\
g_{u}(u, \lambda ; \alpha)=0 \\
g_{\lambda}(u, \lambda ; \alpha)=0 \\
\delta[u-U] \geq 0 ;
\end{array}\right. \\
& \text { (BI) : }\left\{\begin{array}{c}
g(U, \lambda ; \alpha)=0, \\
g_{\lambda}(U, \lambda ; \alpha)=0 ;
\end{array}\right. \\
& (\mathrm{H}):\left\{\begin{array}{c}
g(u, \lambda ; \alpha)=0, \\
g_{u}(u, \lambda ; \alpha)=0, \\
g_{u u}(u, \lambda ; \alpha)=0 \\
\delta[u-U] \geq 0
\end{array}\right. \\
& (\mathrm{HI}):\left\{\begin{array}{l}
g(U, \lambda ; \alpha)=0, \\
g_{u}(U, \lambda ; \alpha)=0,
\end{array}\right. \\
& (\mathrm{DL}):\left\{\begin{array}{c}
g\left(u_{i}, \lambda ; \alpha\right)=0 \\
g_{u}\left(u_{i}, \lambda ; \alpha\right)=0 \\
u_{1} \neq u_{2} \\
\delta\left[u_{i}-U\right] \geq 0
\end{array}\right. \\
& (\mathrm{DLI}):\left\{\begin{array}{c}
g(U, \lambda ; \alpha)=0, \\
g(u, \lambda ; \alpha)=g_{u}(u, \lambda ; \alpha)=0, \\
u \neq U, \delta[u-U] \geq 0,
\end{array}\right.
\end{aligned}
$$

where $\mathrm{B}, \mathrm{H}$, and $\mathrm{DL}$ are nonconstrained bifurcation point set, hysteresis point set, and double limit point set, respectively, and BI, HI, and DLI are constrained bifurcation point set, hysteresis point set, and double limit point set, respectively. Compared with nonconstrained bifurcation $g(u, \lambda ; \alpha)=0$, there exist new transiton sets which are BI, HI, and DLI. For the restriction, there come new bifurcation types, which give the system more bifurcation properties and can explain some nonlinear aspects in engineering systems and other nonlinear systems.

\section{Singularity and Bifurcation Analysis Results}

4.1. Constrained Transition Set. The external current $I$ is chosen as bifurcation parameter and $g_{\mathrm{K}}, g_{L}$ as unfolding parameters, and we restrict $V>0$. It is impossible to give the $\mathrm{HH}$ model's analytic solutions. So, we use the singularity theory of bifurcations and the computing method of bifurcations with constraint in Section 3 to numerically construct the bifurcation diagrams; constraint transition sets are obtained in Figure 1.

From Figure 1 we can conclude that the transition sets without constraint contain only hysteresis set which divides the parameter-plane into two regions, where there are two 


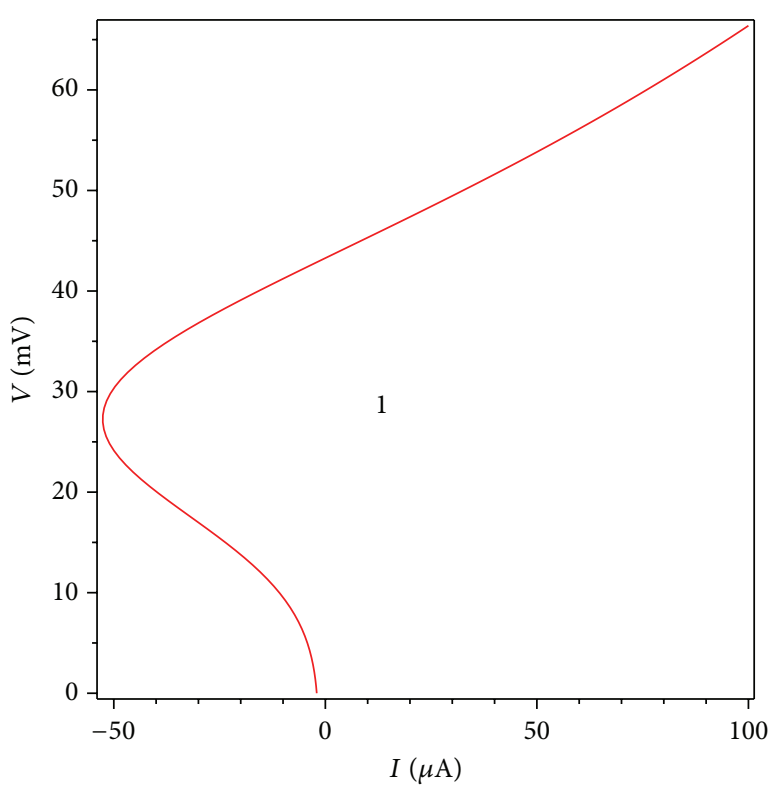

(a) $g_{L}=0.1, g_{\mathrm{K}}=2$

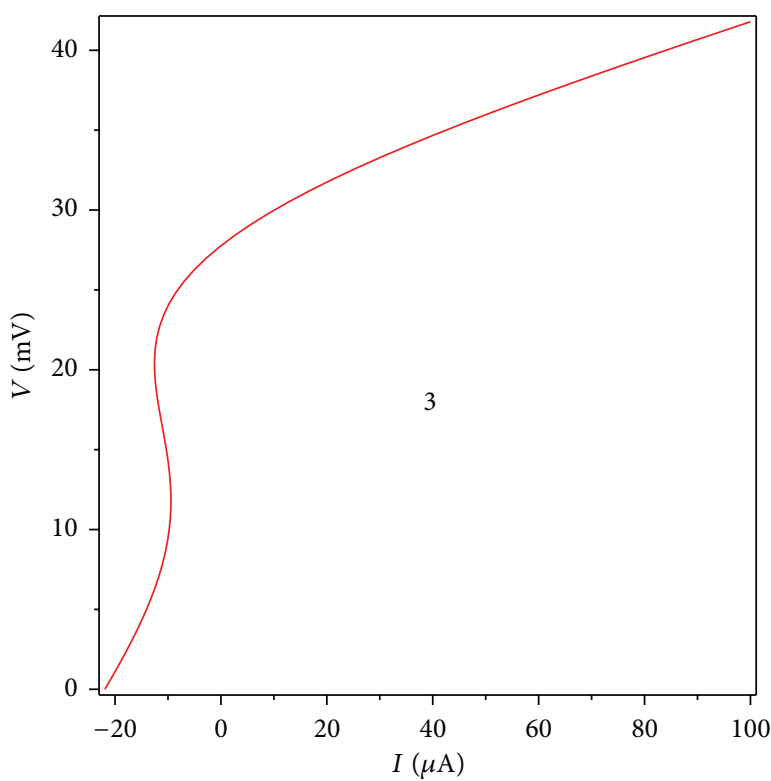

(c) $g_{L}=2, g_{\mathrm{K}}=4$

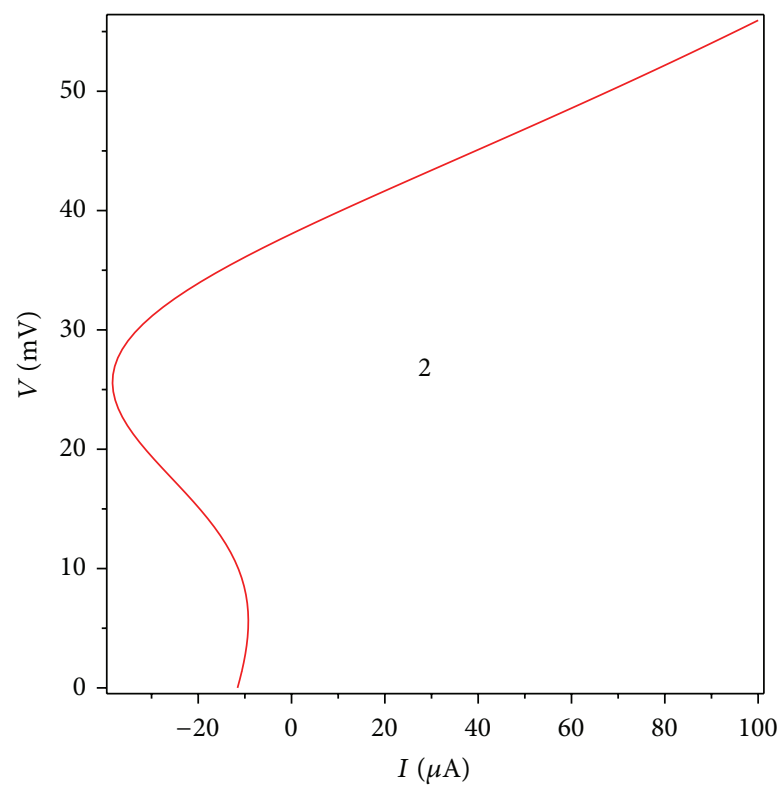

(b) $g_{L}=1, g_{\mathrm{K}}=2$

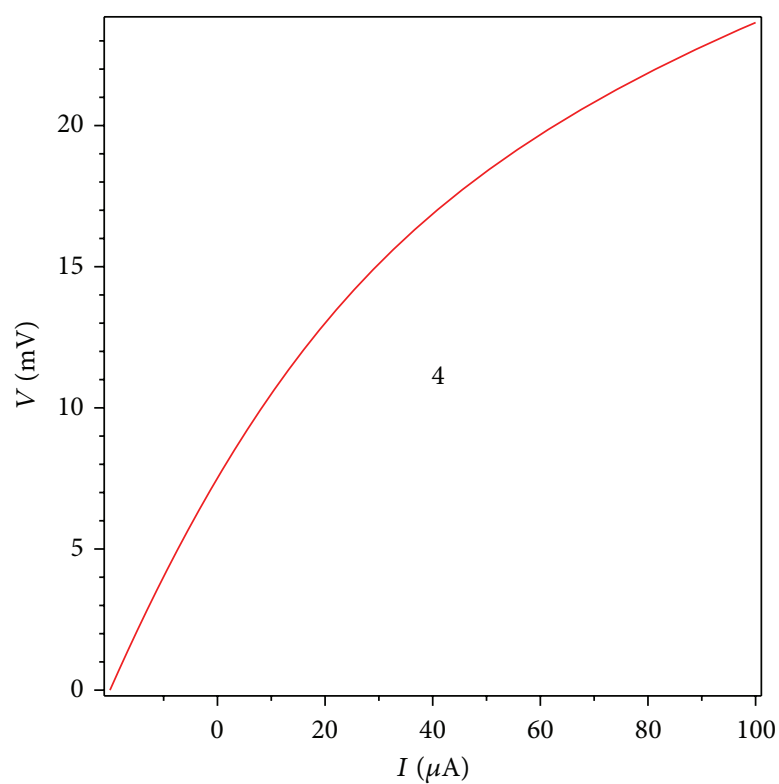

(d) $g_{L}=2, g_{K}=20$

FIgURE 2: The bifurcation diagram for $g_{L}, g_{\mathrm{K}}$ variation.

bifurcation modes. However, the transition sets with constraint contain hysteresis set and double limit set which divide the parameter-plane into four regions, where there are four bifurcation modes. The bifurcation diagram corresponding to four different $g_{L}, g_{\mathrm{K}}$ variations taken from the above four parameters regions is obtained in Figure 2.

\subsection{Bifurcation Analysis Results}

4.2.1. $g_{L}=2, g_{K}=4$. In order to show the bifurcation characteristics of $\mathrm{HH}$, it is convenient to show the bifurcation diagrams obtained by the Matcont software for the varying values of $g_{L}, g_{\mathrm{K}}$. These are given in Figure 3(a). Using the results in Figure 3(a), the stability of equilibrium points is obtained in Figure 3(b). The solid curve denotes the equilibrium points are stable, while the dashed curve denotes the equilibrium points are unstable. Contrasting Figure 3(a) with Figure 2(c), they are identical; then it proves the validity of computing. This can be considered as a verification of the Matcont algorithms for a high order nonlinear system.

Beginning from the left side of the abscissa of Figure 3(a), the first label $\mathrm{H}$ denotes that the equilibrium point is a Hopf bifurcation point with $V=7.609434, m=0.123894, h=$ $0.331941, n=0.437840, I=-11.231605$, and first Lyapunov 


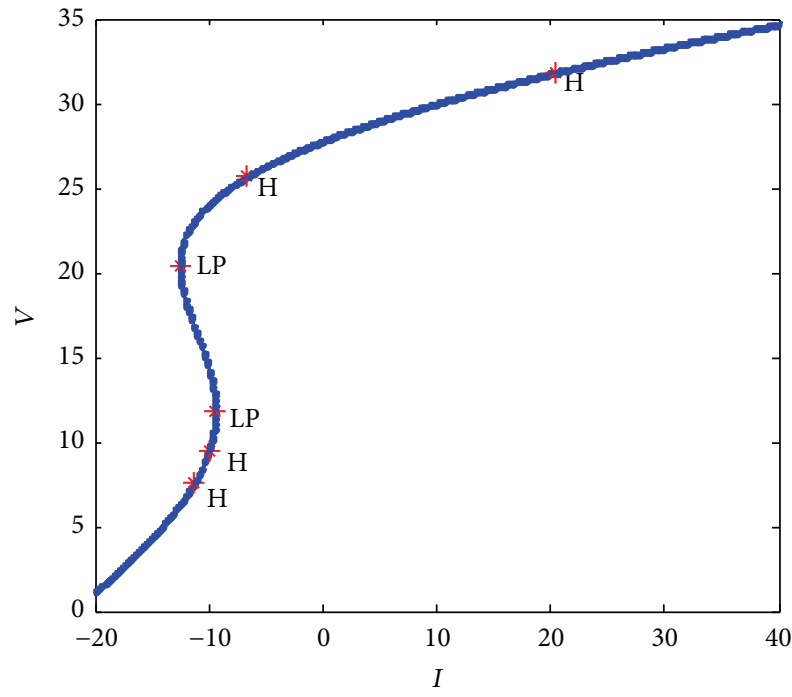

(a) Bifurcation diagram for $I$ variation

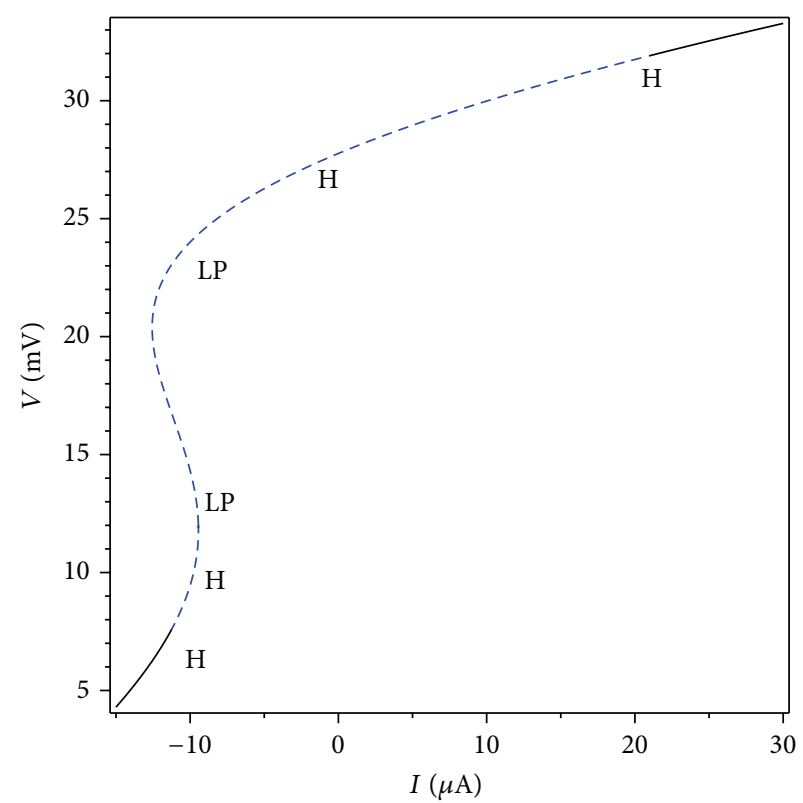

(b) Stability of equilibrium points

FIGURE 3: The bifurcation diagram and stability of equilibrium points for $g_{L}=2, g_{\mathrm{K}}=4$.

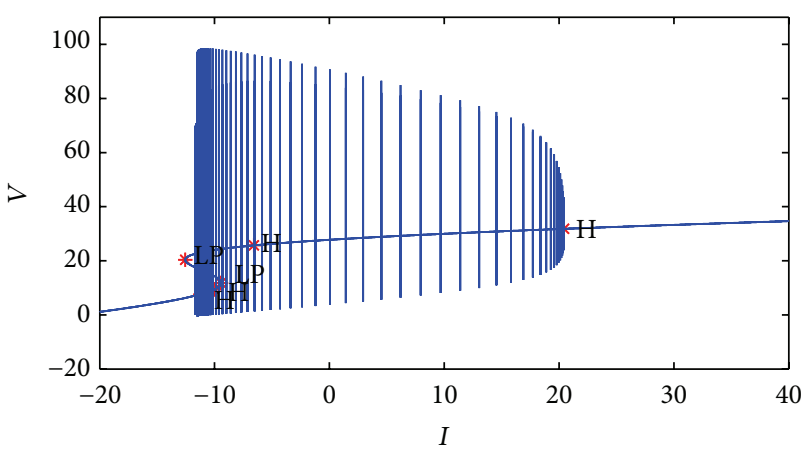

Figure 4: The limit cycle emerging from sH at $I=20.428518$.

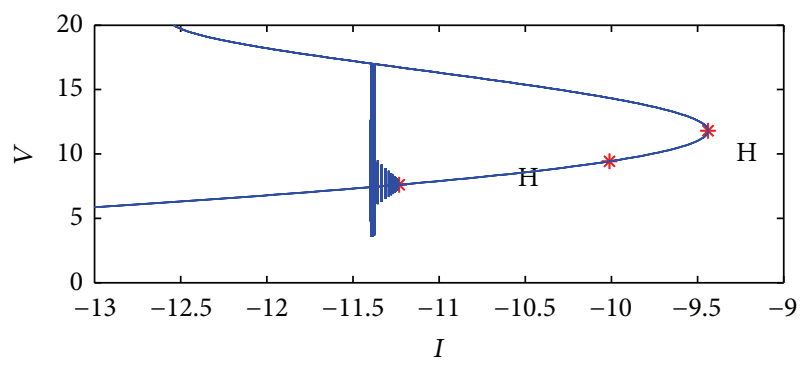

FIgURE 5: The limit cycle emerging from sH at $I=-11.231605$.

coefficient is positive, and there are two eigenvalues with $\operatorname{Re} \lambda_{1,2} \approx 0, \operatorname{Im} \lambda_{1} \neq 0$; then at the Hopf bifurcation point, $\mathrm{HH}$ is unstable, and there is an unstable limit cycle, so it is the subcritical Hopf bifurcation ( $\mathrm{uH}$ ). Although the equilibrium points of the second and fifth are labelled as $\mathrm{H}$, they are not Hopf bifurcation points. They are neutral saddle point, where
TABLE 1: Bifurcation analysis results derived by the Matcont software.

\begin{tabular}{|c|c|c|c|}
\hline Parameter & $\begin{array}{l}\text { Equilibrium } \\
\text { points }\end{array}$ & $I$ & Type of condition \\
\hline \multirow{6}{*}{$\begin{array}{l}g_{L}=2 \\
g_{K}=4\end{array}$} & $\begin{aligned} V & =7.609434 \\
m & =0.123894 \\
h & =0.331941 \\
n & =0.437840\end{aligned}$ & $I=-11.231605$ & $\mathrm{uH}$ \\
\hline & $\begin{aligned} V & =9.426017 \\
m & =0.149260 \\
h & =0.278301 \\
n & =0.466512\end{aligned}$ & $I=-10.010747$ & Neutral saddle \\
\hline & $\begin{array}{c}V=25.688959 \\
m=0.518771 \\
h=0.046889 \\
n=0.686086\end{array}$ & $I=-6.575754$ & Neutral saddle \\
\hline & $\begin{array}{c}V=11.796299 \\
m=0.188048 \\
h=0.217790 \\
n=0.503195\end{array}$ & $I=-9.438630$ & Limit point \\
\hline & $\begin{array}{c}V=20.373201 \\
m=0.378788 \\
h=0.083800 \\
n=0.623808\end{array}$ & $I=-12.559365$ & Limit point \\
\hline & $\begin{array}{c}V=31.816299 \\
m=0.668810 \\
h=0.025491 \\
n=0.745429\end{array}$ & $I=20.428518$ & $\mathrm{sH}$ \\
\hline
\end{tabular}

the former has $V=9.4260170, m=0.149260, h=0.278301$, $n=0.466512, I=-10.010747$, and the latter has $V=$ 


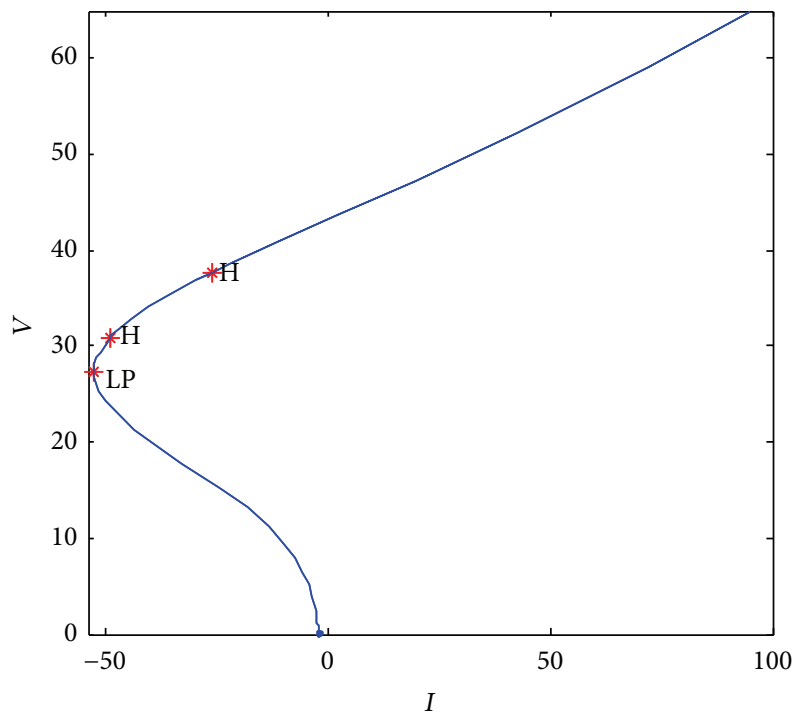

(a) Bifurcation diagram for $I$ variation

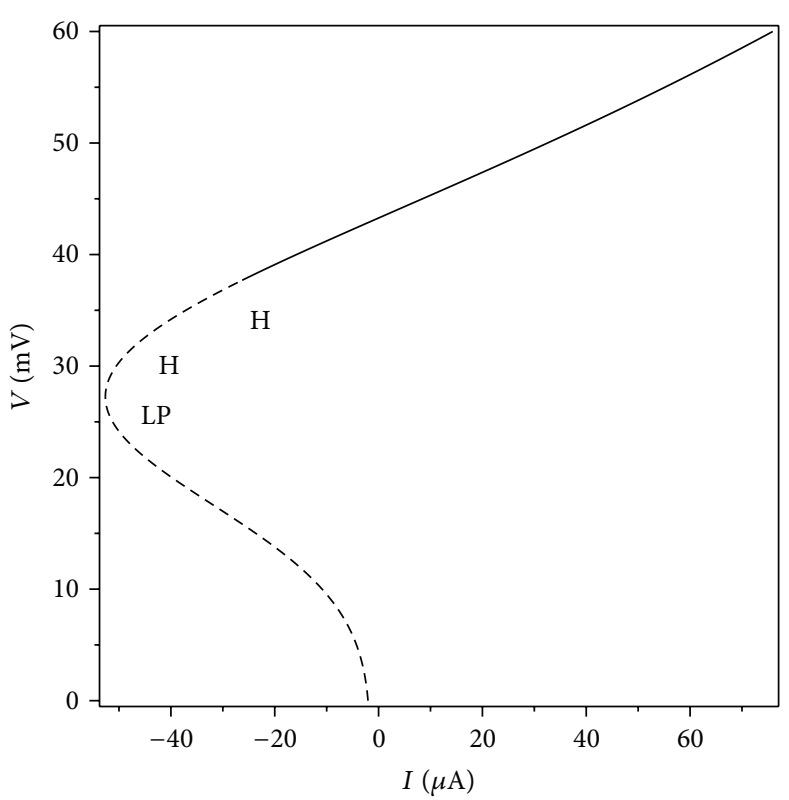

(b) Stability of equilibrium points

FIGURE 6: The bifurcation diagram and stability of equilibrium points for $g_{L}=0.1, g_{\mathrm{K}}=2$.

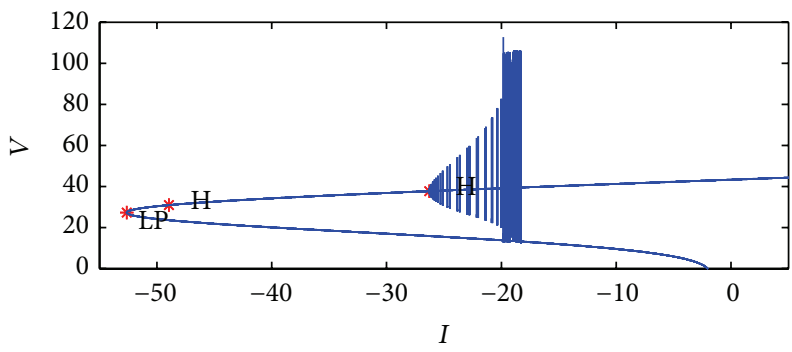

Figure 7: The limit cycle emerging from uH at $I=-26.281425$.

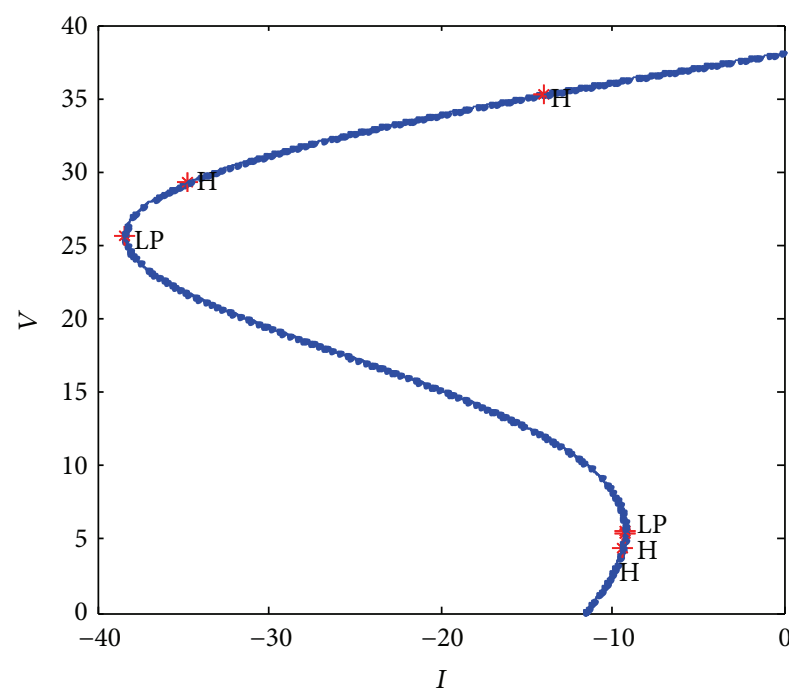

(a) Bifurcation diagram for $I$ variation

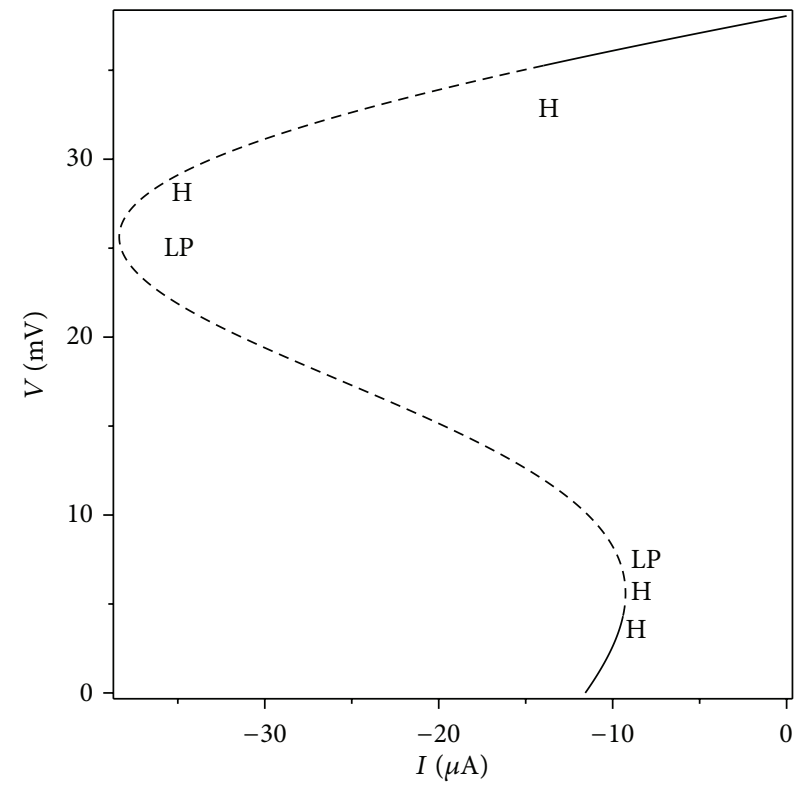

(b) Stability of equilibrium points

FIgURE 8: The bifurcation diagram and stability of equilibrium for $g_{L}=1, g_{\mathrm{K}}=2$. 


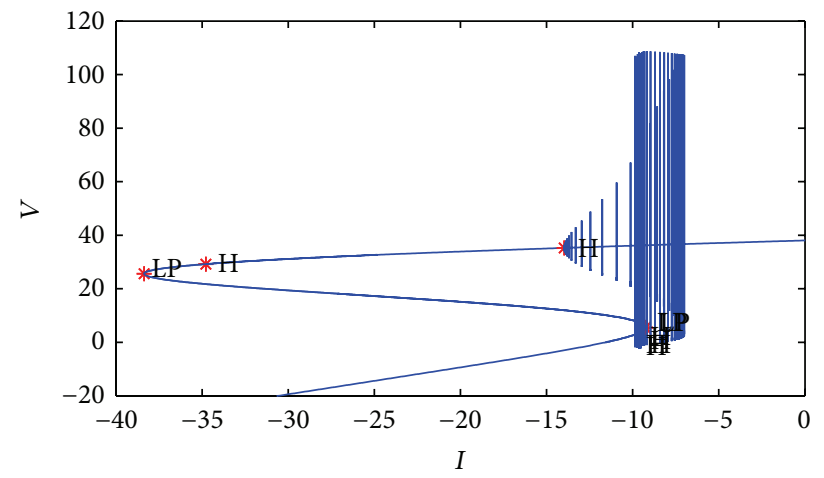

Figure 9: The limit cycle emerging from $\mathrm{uH}$ at $I=-13.971904$.

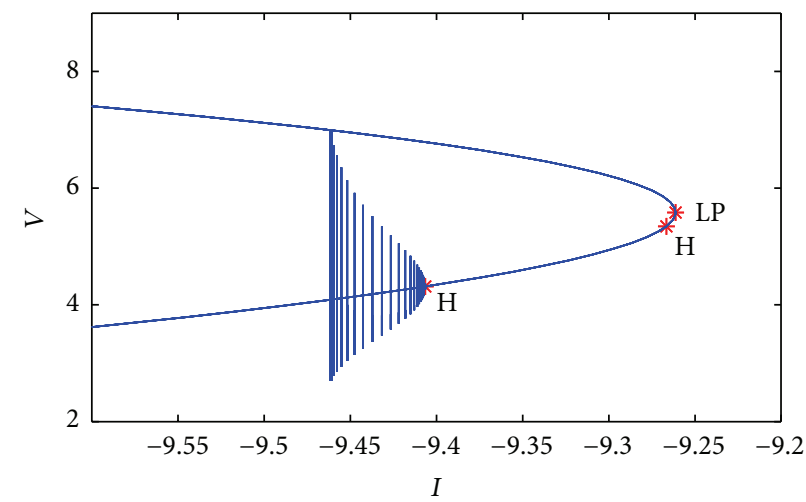

Figure 10: The limit cycle emerging from $\mathrm{uH}$ at $I=-9.406580$.

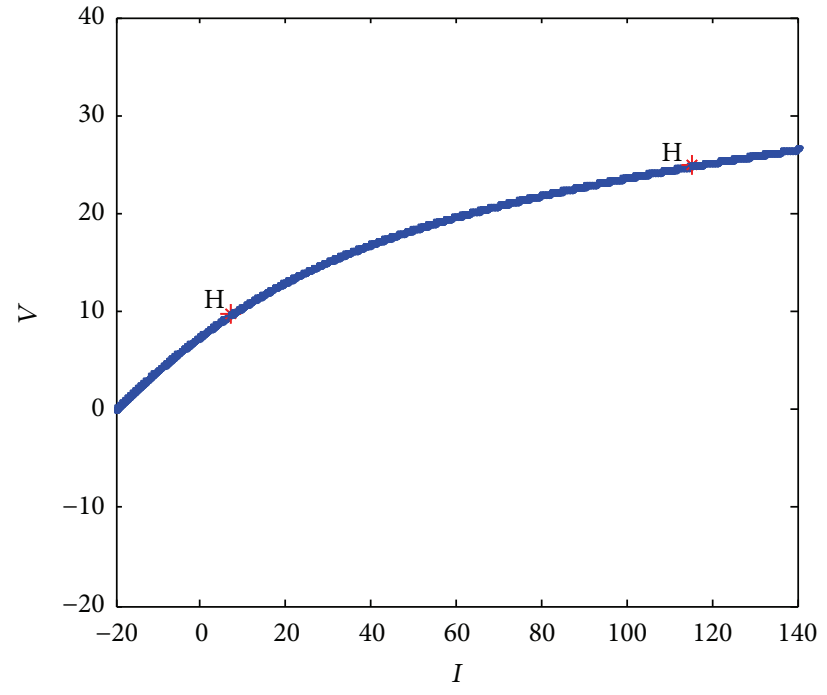

(a) Bifurcation diagram for $I$ variation

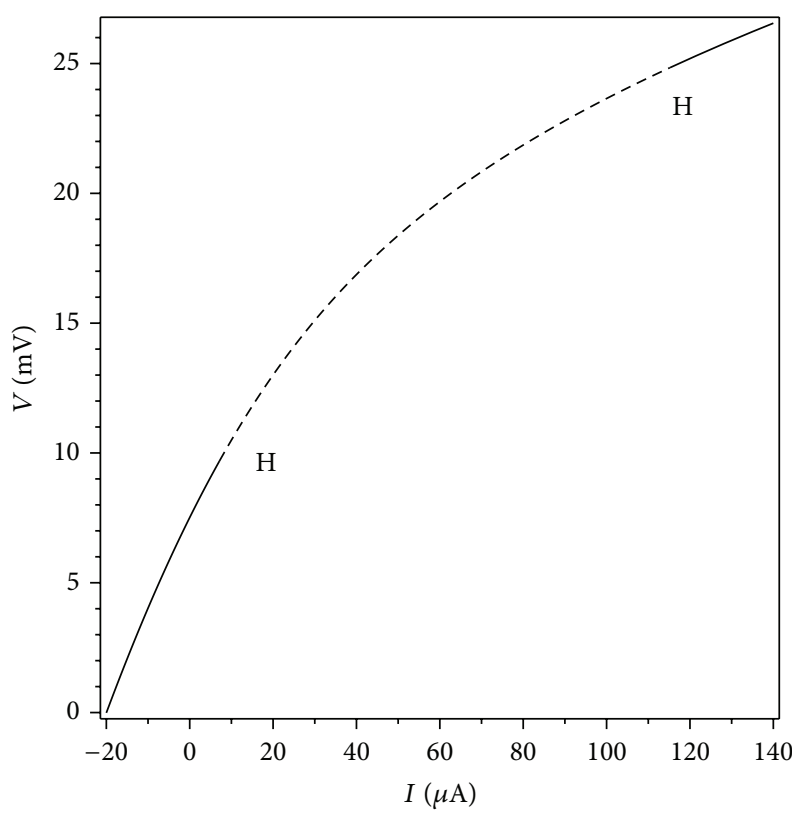

(b) Stability of equilibrium

FIGURE 11: The bifurcation diagram and stability of equilibrium point for $g_{L}=2, g_{\mathrm{K}}=20$. 


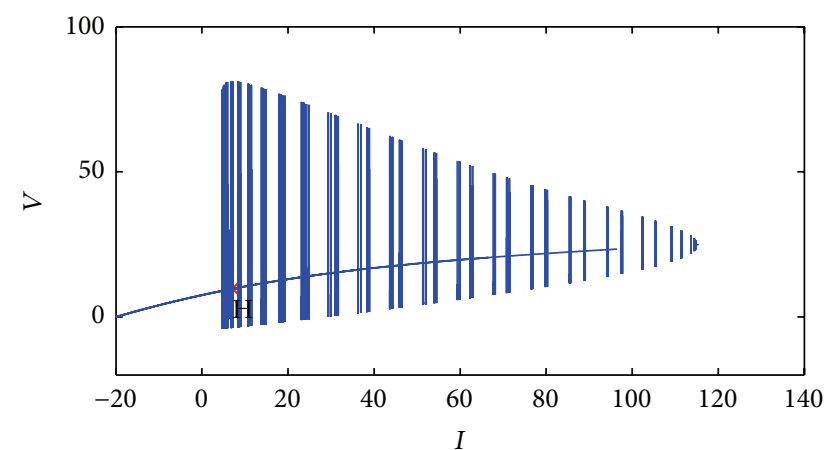

Figure 12: The limit cycle emerging from $\mathrm{uH}$ at $I=7.131765$ and $\mathrm{sH}$ at $I=115.224276$.

TABLE 2: Bifurcation analysis results derived by the Matcont software.

\begin{tabular}{|c|c|c|c|}
\hline Parameter & $\begin{array}{l}\text { Equilibrium } \\
\text { points }\end{array}$ & $I$ & Type of condition \\
\hline \multirow{6}{*}{$\begin{array}{l}g_{L}=0.1 \\
g_{K}=2\end{array}$} & $\begin{array}{c}V=-3.692856 \\
m=0.033952 \\
h=0.716772 \\
n=0.262913\end{array}$ & $I=-1.749367$ & Limit point \\
\hline & $\begin{array}{c}V=-0.754488 \\
m=0.048408 \\
h=0.622236 \\
n=0.306181\end{array}$ & $I=-1.918161$ & Neutral saddle \\
\hline & $\begin{array}{c}V=-1.822633 \\
m=0.042603 \\
h=0.658089 \\
n=0.290154\end{array}$ & $I=-1.811258$ & Neutral saddle \\
\hline & $\begin{array}{c}V=27.252348 \\
m=0.559277 \\
h=0.039851 \\
n=0.702462\end{array}$ & $I=-52.626190$ & Limit point \\
\hline & $\begin{array}{c}V=30.900097 \\
m=0.648151 \\
h=0.027784 \\
n=0.737363\end{array}$ & $I=-48.954977$ & Neutral saddle \\
\hline & $\begin{array}{c}V=37.692334 \\
m=0.781785 \\
h=0.015320 \\
n=0.791063\end{array}$ & $I=-26.281425$ & $\mathrm{uH}$ \\
\hline
\end{tabular}

25.688959, $m=0.518771, h=0.046889, n=0.686086$, $I=-6.575754$. The equilibrium points labelled as LP of the third and fourth are both limit points, where the former has $V=11.796299, m=0.188048, h=0.217790, n=0.503195$, $I=-9.438630$, and the latter has $V=20.373201, m=$ $0.378788, h=0.083800, n=0.623808, I=-12.559365$. The equilibrium point labelled as $\mathrm{H}$ of the sixth is a Hopf bifurcation point with $V=31.816299, m=0.668810, h=$ $0.025491, n=0.745429, I=20.428518$, and first Lyapunov coefficient is negative, and there are two eigenvalues with $\operatorname{Re} \lambda_{1,2} \approx 0$; then at the Hopf bifurcation point, $\mathrm{HH}$ is stable, and there is a stable limit cycle, so it is the supercritical Hopf
TABLE 3: Bifurcation analysis results derived by the Matcont software.

\begin{tabular}{|c|c|c|c|}
\hline Parameter & $\begin{array}{l}\text { Equilibrium } \\
\text { points }\end{array}$ & $I$ & Type of condition \\
\hline \multirow{6}{*}{$\begin{array}{l}g_{L}=1 \\
g_{K}=2\end{array}$} & $\begin{aligned} V & =4.315751 \\
m & =0.086823 \\
h & =0.442071 \\
n & =0.385365\end{aligned}$ & $I=-9.406580$ & $\mathrm{uH}$ \\
\hline & $\begin{aligned} V & =5.346944 \\
m & =0.097269 \\
h & =0.406190 \\
n & =0.401801\end{aligned}$ & $I=-9.266520$ & Neutral saddle \\
\hline & $\begin{aligned} V & =5.583327 \\
m & =0.099807 \\
h & =0.398116 \\
n & =0.405572\end{aligned}$ & $I=-9.261244$ & Limit point \\
\hline & $\begin{array}{c}V=25.615559 \\
m=0.516847 \\
h=0.047253 \\
n=0.685295\end{array}$ & $I=-38.368717$ & Limit point \\
\hline & $\begin{array}{c}V=29.221394 \\
m=0.608443 \\
h=0.032689 \\
n=0.721865\end{array}$ & $I=-34.782328$ & Neutral saddle \\
\hline & $\begin{array}{c}V=35.263043 \\
m=0.739314 \\
h=0.018740 \\
n=0.773422\end{array}$ & $I=-13.971904$ & $\mathrm{uH}$ \\
\hline
\end{tabular}

bifurcation (sH). Equilibrium points between the first $\mathrm{H}$ and the sixth $\mathrm{H}$ are unstable.

The limit cycle emerging from sH at $I=20.428518$ is in Figure 4.

The limit cycle emerging from $\mathrm{uH}$ at $I=-11.231605$ is in Figure 5.

In Table 1, the bifurcation points found by the Matcont software are presented for $g_{L}=2, g_{K}=4$.

4.2.2. $g_{L}=0.1, g_{K}=2$. The bifurcation diagrams and the stability of equilibrium points are obtained in Figure 6. The limit cycle emerging from $\mathrm{uH}$ at $I=-26.281425$ is in Figure 7. The bifurcation points found by the Matcont software are presented in Table 2 for $g_{L}=0.1, g_{\mathrm{K}}=2$.

From Table 2, we can find the difference in equilibrium point.

4.2.3. $g_{L}=1, g_{K}=2$. The bifurcation diagrams and the stability of equilibrium points are obtained in Figure 8 . The limit cycles emerging from $\mathrm{uH}$ at $I=-13.971904$ and $I=-9.406580$ are given in Figures 9 and 10, respectively. The bifurcation points found by the Matcont software are presented in Table 3 for $g_{L}=1, g_{\mathrm{K}}=2$.

4.2.4. $g_{L}=2, g_{K}=20$. The bifurcation diagrams and the stability of equilibrium points are obtained in Figure 11. The limit cycle emerging from $\mathrm{uH}$ at $I=7.131765$ and $\mathrm{sH}$ at 
TABLE 4: Bifurcation analysis results derived by the Matcont software.

\begin{tabular}{lccc}
\hline Parameter & $\begin{array}{c}\text { Equilibrium } \\
\text { points }\end{array}$ & $I$ & Type of condition \\
& $V=9.688168$ & & \\
& $m=0.153228$ & & \\
& $h=0.271065$ & $I=7.131765$ & $\mathrm{uH}$ \\
$g_{L}=2$ & $n=0.470616$ & & \\
$g_{\mathrm{K}}=20$ & $V=24.842866$ & & \\
& $m=0.496500$ & & $\mathrm{sH}$ \\
& $h=0.051294$ & $I=115.224276$ & \\
& $n=0.676858$ & & \\
\hline
\end{tabular}

$I=115.224276$ is given in Figure 12. They both are the same. The bifurcation points found by the Matcont software are presented in Table 4 for $g_{L}=2, g_{K}=20$.

\section{Conclusion}

In this paper we present the equilibrium point bifurcation and singularity analysis of $\mathrm{HH}$ model with constraints. We investigate the effect of constraints and parameters on the type of equilibrium point bifurcation. We find that if we restrict $V>0$, then there are new transition sets, and new bifurcation type constrating to the nonconstraint case. The Matcont toolbox software environment was used for analysis of the bifurcation points in conjunction with Matlab. We give four different parameters of $g_{L}, g_{\mathrm{K}}$. In each case, we give equilibrium point bifurcation and also illustrate the stability of the equilibrium points. This study increases our knowledge of $\mathrm{HH}$ model.

\section{Conflict of Interests}

The authors declare that there is no conflict of interests regarding the publication of this paper.

\section{Acknowledgments}

This study is supported by the National Science Foundation of China (Grant no. 11172198) and the National Basic Research Program of China (Grant no. 11372211).

\section{References}

[1] A. L. Hodgkin and A. F. Huxley, "A quantitative description of membrane current and its application to conduction and excitation in nerve.," The Journal of Physiology, vol. 117, no. 4, pp. 500-544, 1952.

[2] R. Fitzhugh, "Mathematical models of excitation and propagation in nerve," in Biological Engineer, H. P. Schwan, Ed., McGraw-Hill, New York, NY, USA, 1969.

[3] J. Cronin, Mathematical Aspects of Hodgkin-HUXley Neural Theory, Cambridge University Press, 1987.

[4] T. R. L. Chay and J. Keizer, "Minimal model for membrane oscillations in the pancreatic $\beta$-cell," Biophysical Journal, vol. 42, no. 2, pp. 181-189, 1983.
[5] J. Keener and J. Sneyd, Mathematical Physiology, vol. 8 of Interdisciplinary Applied Mathematics, Springer, New York, NY, USA, 1998.

[6] J. Guckenheimer and P. Holmes, Nonlinear Oscillations, Dynamical Systems, and Bifurcation of Vector Fields, Springer, New York, NY, USA, 1983.

[7] J. Guckenheimer and J. S. Labouriau, "Bifurcation of the Hodgkin and Huxley equations: a new twist," Bulletin of Mathematical Biology, vol. 55, no. 5, pp. 937-952, 1993.

[8] Y. A. Bedrov, G. N. Akoev, and O. E. Dick, "Partition of the Hodgkin-Huxley type model parameter space into the regions of qualitatively different solutions," Biological Cybernetics, vol. 66, no. 5, pp. 413-418, 1992.

[9] Y. A. Bedrov, O. E. Dick, A. D. Nozdrachev, and G. N. Akoev, "Method for constructing the boundary of the bursting oscillations region in the neuron model," Biological Cybernetics, vol. 82, no. 6, pp. 493-497, 2000.

[10] H. Fukai, S. Doi, T. Nomura, and S. Sato, "Hopf bifurcations in multiple-parameter space of the Hodgkin-Huxley equations I. Global organization of bistable periodic solutions," Biological Cybernetics, vol. 82, no. 3, pp. 215-222, 2000.

[11] H. Fukai, T. Nomura, S. Doi, and S. Sato, "Hopf bifurcations in multiple-parameter space of the Hodgkin-Huxley equations II. Singularity theoretic approach and highly degenerate bifurcations," Biological Cybernetics, vol. 82, no. 3, pp. 223-229, 2000.

[12] M. Golubitsky and D. G. Schaeffer, Singularities and Groups in Bifurcation Theory. Vol. I, vol. 51 of Applied Mathematical Sciences, Springer, New York, NY, USA, 1985.

[13] Z. Q. Wu and Y. S. Chen, "Classification of bifurcations for nonlinear dynamical problems with constraints," Applied Mathematics and Mechanics, vol. 23, no. 5, pp. 535-541, 2002. 


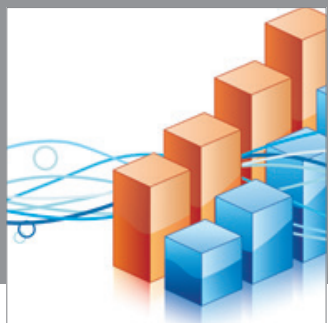

Advances in

Operations Research

mansans

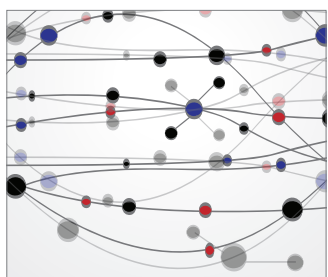

The Scientific World Journal
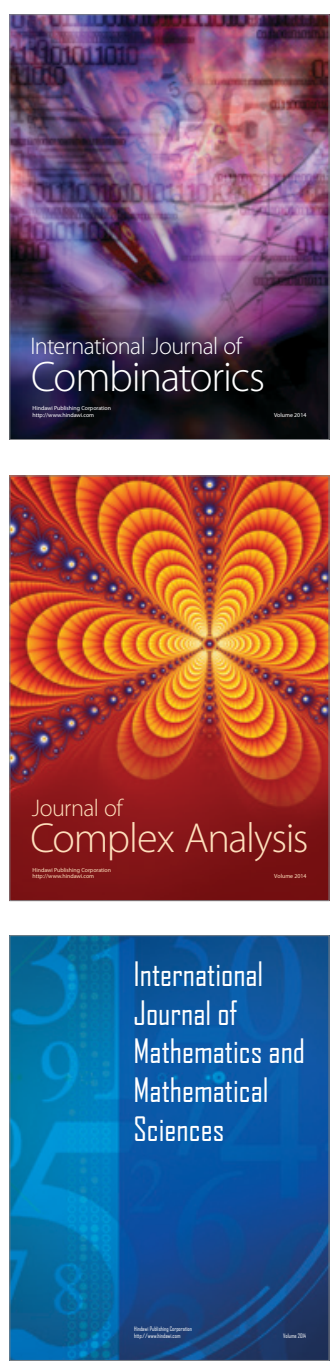
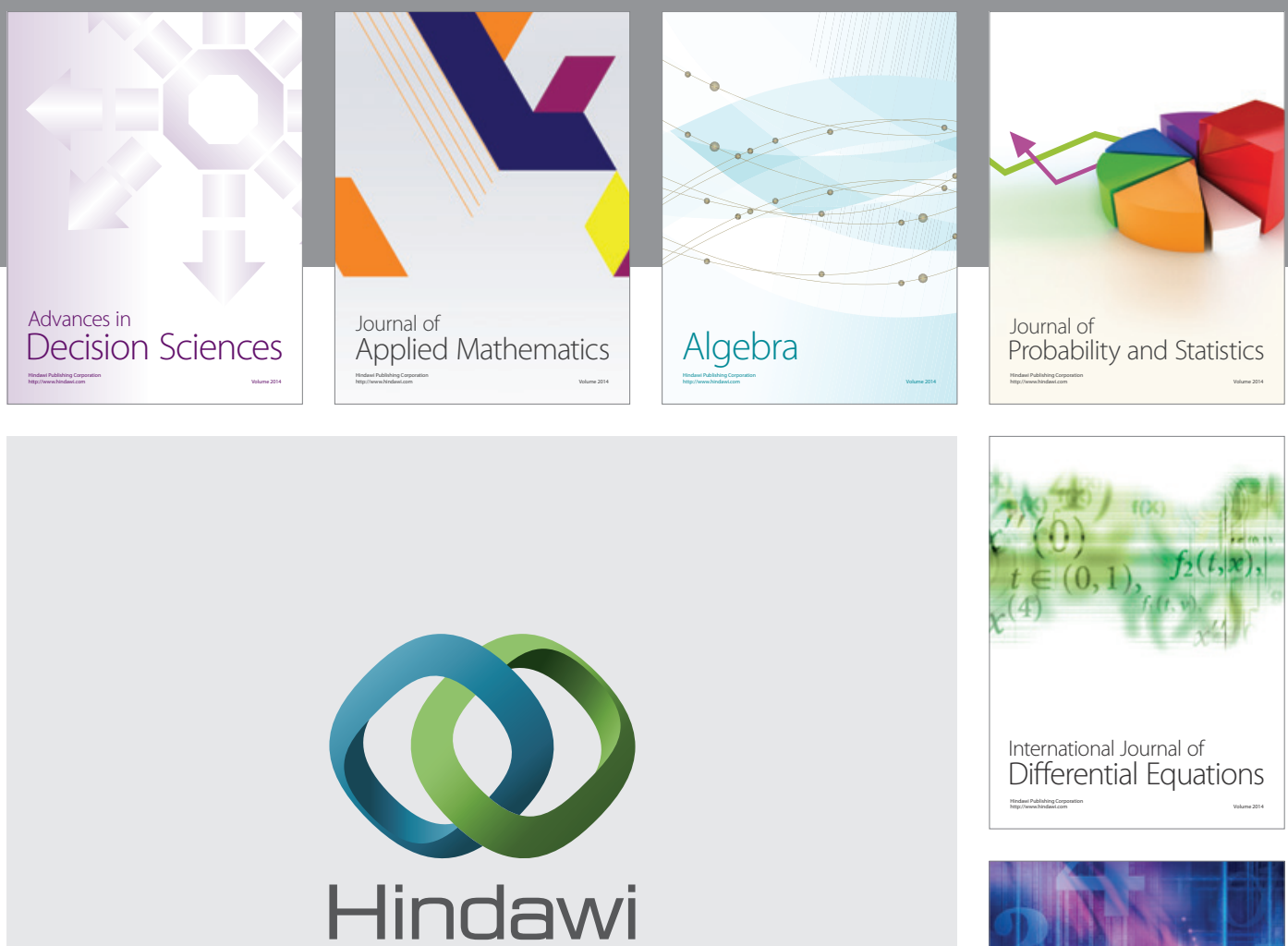

Submit your manuscripts at http://www.hindawi.com
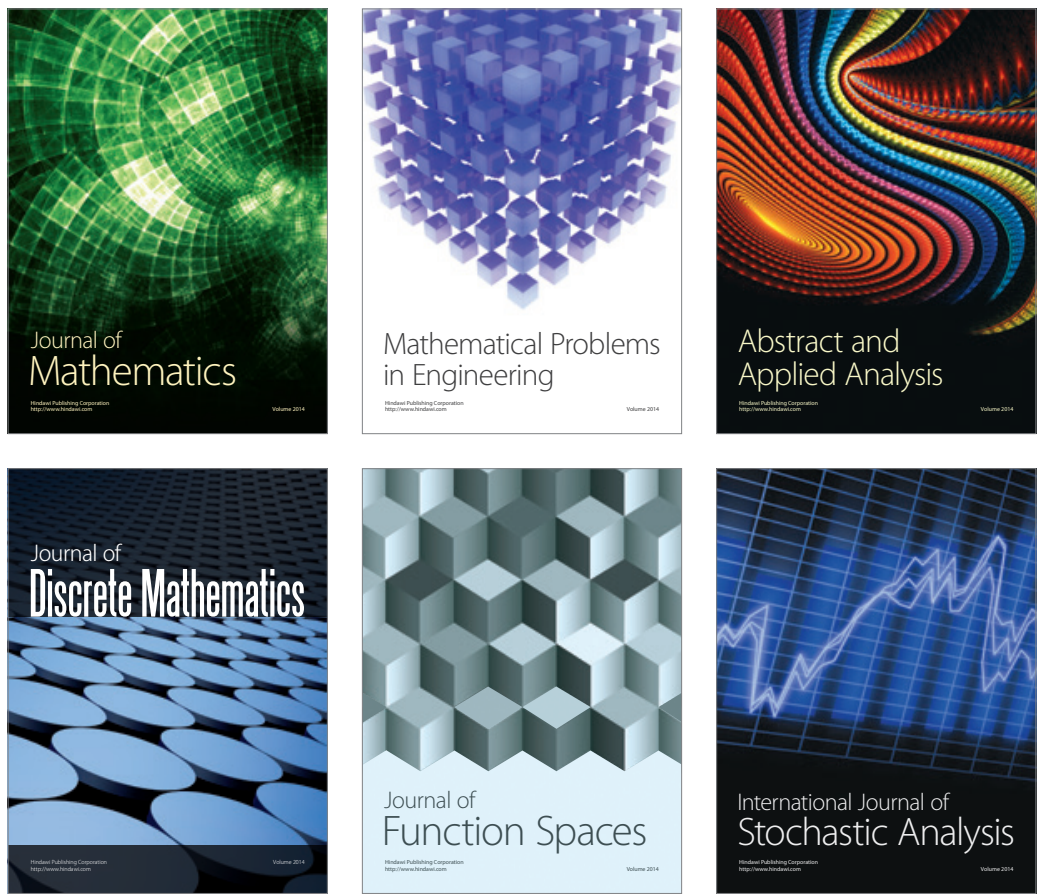

Journal of

Function Spaces

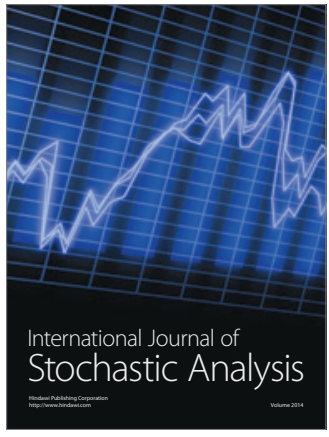

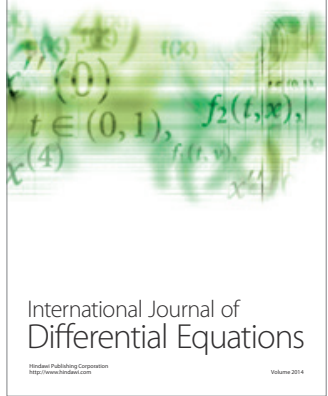
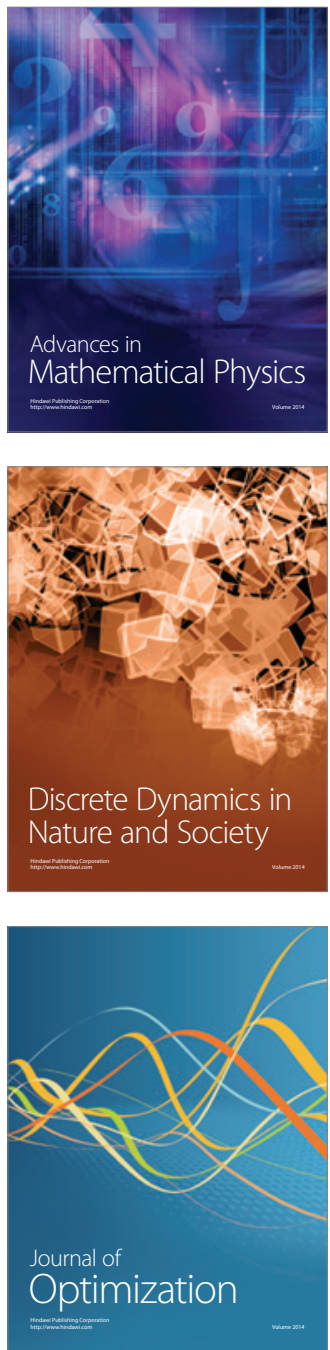